\title{
A Tighter Test of the Local Lorentz Invariance of Gravity Using PSR J2317+1439
}

\author{
J. F. Bell \\ Mount Stromlo and Siding Spring Observatories, Institute of Advanced Studies, \\ Australian National University, Private Bag, Weston Creek, ACT 2611, Australia \\ email: bell@mso.anu.edu.au \\ F. Camilo \\ Joseph Henry Laboratories and Physics Department, \\ Princeton University, Princeton, NJ 08544 \\ and \\ University of Manchester, NRAL, Jodrell Bank, Macclesfield, Cheshire SK11 9DL, UK \\ email: fernando@pulsar.princeton.edu \\ T. Damour \\ Institut des Hautes Etudes Scientifiques, F-91440 Bures-sur-Yvette, France \\ and \\ Département d'Astrophysique Relativiste et de Cosmologie, Observatoire de Paris, \\ Centre National de la Recherche Scientifique, F-92195 Meudon, France \\ email:damour@ihes.fr
}

\begin{abstract}
Gravity being a long-range force, one might a priori expect the Universe's global matter distribution to select a preferred rest frame for local gravitational physics. The phenomenology of preferred-frame effects, in the strong-gravitational field context of binary pulsars, is described by two parameters $\hat{\alpha}_{1}$ and $\hat{\alpha}_{2}$. These parameters vanish identically in general relativity, and reduce, in the weak-field limit, to the two parametrized post-Newtonian (PPN) parameters $\alpha_{1}$ and $\alpha_{2}$. We derive a limit of $\left|\hat{\alpha}_{1}\right|<1.7 \times 10^{-4}$ (90\% C.L.) using the very low eccentricity binary pulsar PSR J2317+1439, improving by a factor of 3 on previous limits.
\end{abstract}

Subject headings: gravitation — relativity — pulsars: individual (PSR J2317+1439) 
General relativity might not be the last word on gravity. On the one hand, we do not know how to quantize it, and on the other hand, modern unification theories suggest that the low-energy limit of unified theories might contain, besides the tensor field postulated by Einstein, other long-range fields participating in the gravitational interaction between macroscopic bodies. The present paper follows and improves upon previous results by showing how binary pulsar data can set very tight limits on such possible long-range deviations from general relativity.

In the weak-field context appropriate to describing the solar system the parametrized postNewtonian (PPN) formalism is used as a tool for analyzing gravitation theory and experiment. It provides a set of parameters (the PPN parameters) which take different values in different theories and can be related to measurable quantities, forming a basis for comparison of theory and experiment. See Will (1993) for a summary of the PPN formalism, PPN parameters, and the values they take in various theories of gravity. In theories where gravity is mediated in part by a longrange vector field (or a second tensor field, besides the one postulated by Einstein), one expects the Universe's global matter distribution to select a preferred rest frame for local gravitational physics (violation of local Lorentz invariance). In the post-Newtonian limit the phenomenology of preferred-frame effects is describable by two PPN parameters $\alpha_{1}$ and $\alpha_{2}$. Gravitation theories, such as general relativity, which respect local Lorentz invariance (absence of a preferred frame) have parameters $\alpha_{1} \equiv \alpha_{2} \equiv 0$. These two parameters have been constrained using solar-system data $\left|\alpha_{2}\right|<3.9 \times 10^{-7}, \alpha_{1}=(2.1 \pm 3.1) \times 10^{-4}$ (90\% C.L.; Nordtvedt 1987; Hellings 1984; Will 1993).

Binary pulsars take us beyond the weak-field context because the self gravitational field of a neutron star is very strong. This strength is conveniently measured by the "compactness" parameter $c_{A}=-2 \partial \ln m_{A} / \partial \ln G$, with value $c_{1} \simeq 0.3$ when $m_{1} \simeq 1.4 \mathrm{M}_{\odot}$ (Damour \& Esposito-Farèse 1992a). To discuss tests of gravity using binary pulsar data, Damour and Taylor (1992) introduced the parametrized post-Keplerian (PPK) formalism in which a pulsar timing experiment is parametrized by a set of phenomenological parameters which lump together all possible strongfield effects. The original PPK formalism (see also Damour \& Schäfer 1991) did not allow for preferred-frame effects. Damour and Esposito-Farèse (1992b), hereafter DE92b, extended the PPK formalism by adding strong-field generalizations of the usual PPN preferred-frame parameters, and showed how certain low-eccentricity binary systems provided excellent testing grounds for possible violations of local Lorentz invariance. Their parameters $\hat{\alpha}_{1}, \hat{\alpha}_{2}$, are conceived as including the weak field contribution $\alpha_{1}, \alpha_{2}$, plus possible strong-field modifications proportional to successive powers of the compactnesses of the two stars: $\hat{\alpha}_{1}=\alpha_{1}+\alpha_{1}^{\prime}\left(c_{1}+c_{2}\right)+\cdots$; ; a similar relation applies for $\alpha_{2}$. In general relativity $\hat{\alpha}_{1} \equiv 0 \equiv \hat{\alpha}_{2}$, while these parameters may be different from zero in theories where gravity is partially mediated by a vector or a second tensor field. Binary pulsar data have already given a good limit on $\hat{\alpha}_{1}$ : $\left|\hat{\alpha}_{1}\right|<5.0 \times 10^{-4}$ (90\% C.L.) (DE92b). Following the methodology of DE92b, we shall show here how the use of the data on the very low-eccentricity binary pulsar J2317+1439 allows one to significantly tighten this limit. 
If $\hat{\alpha}_{1} \neq 0$ a consequence is a constant forcing term in the time evolution of the eccentricity vector of a binary stellar system. For a very low eccentricity orbit, this tends to "polarize" the orbit, aligning the eccentricity vector with the projection onto the orbital plane of the absolute velocity of the system. Hence, the orbital parameters of very low eccentricity binary pulsars may be used to set an upper bound on $\left|\hat{\alpha}_{1}\right|$ (DE92b), given by

$$
\left|\hat{\alpha}_{1}\right|<(10 / \pi) I_{i, \lambda} e / \hat{e} \quad(90 \% \text { C.L. })
$$

where

$$
I_{i, \lambda}=\int_{0}^{2 \pi} \frac{(2 \pi)^{-1} d \Omega}{\sqrt{1-(\cos i \cos \lambda+\sin i \sin \lambda \sin \Omega)^{2}}}
$$

and

$$
\hat{e}=\frac{1}{12}\left|\frac{m_{1}-m_{2}}{m_{1}+m_{2}}\right| \frac{\left|\mathbf{w}_{\mathrm{psr}}\right|}{\left(G\left(m_{1}+m_{2}\right) 2 \pi / P_{b}\right)^{1 / 3}} .
$$

In equations 1 $3, e$ is the orbital eccentricity, $\Omega$ is the longitude of the node of the binary orbit with respect to the line of sight, $i$ is the orbital inclination, $\mathbf{w}_{\mathrm{psr}}$ is the absolute velocity of the center of mass of the binary system, $\lambda$ is the angle between $\mathbf{w}_{\mathrm{psr}}$ and the line of sight, $m_{1}$ is the pulsar mass, $m_{2}$ is the companion mass, $G$ is the gravitational constant, and $P_{b}$ is the orbital period.

The very low eccentricity binary pulsar J2317+1439 (Camilo, Nice, \& Taylor 1993; Camilo, Nice, \& Taylor 1995) has a figure of merit for the determination of limits on $\hat{\alpha}_{1}$ (DE92b), 10 times better than PSR B1855+09 which was used to obtain the previous best limit. Hence PSR J2317+1439 should provide a substantially tighter limit on $\left|\hat{\alpha}_{1}\right|$. To obtain the magnitude of the absolute velocity $\left|\mathbf{w}_{\mathrm{psr}}\right|$, the cosmic microwave background is chosen as the preferred reference frame. The results from the Cosmic Background Explorer (Smoot et al. 1991; Fixsen et al. 1994) for motion of the solar system with respect to the cosmic microwave background give $\left|\mathbf{w}_{\odot}\right|=369 \mathrm{~km} \mathrm{~s}^{-1}$ in the direction (R.A., Dec) $=\left(11.3^{\mathrm{h}},-7^{\circ}\right)$. This direction is almost antiparallel to the line of sight to PSR J2317+1439 $\left(\lambda=192^{\circ}\right)$. No estimate of the radial velocity of PSR J2317+1439 is available - however Camilo, Nice, \& Taylor (1995), report a velocity in the plane of the sky of $v_{\perp}=(70 \pm 30) \mathrm{km} \mathrm{s}^{-1}$. Excluding, at the $90 \%$ C.L., an unfavourable alignment within $\theta_{\text {min }}=\arccos (0.9)=25.8^{\circ}$ between $\mathbf{w}_{\odot}$ and minus the (solar barycentric) velocity of the pulsar, gives a firm lower limit $\left|\mathbf{w}_{\mathrm{psr}}\right|>\sqrt{\left(\mathbf{w}_{\odot}-\mathrm{v}_{\perp} \cot \left(\theta_{\min }\right)\right)^{2}+\mathrm{v}_{\perp}^{2}} \simeq 235 \mathrm{~km} \mathrm{~s}^{-1}$. The characteristic age of $23 \times 10^{9} \mathrm{yr}$ for PSR J2317+1439, and the cooling age for its stellar companion of greater than $10^{9}$ yr (Camilo, Nice, \& Taylor 1995), ensure that it has been a long time since mass transfer in the binary system ceased, satisfying the requirement that systems used for this test be old (DE92b).

Radio timing results give $P_{b}=2.459 \mathrm{~d}$ and $e<1.2 \times 10^{-6}$ (90\% C.L.) for PSR J2317+1439 (Camilo, Nice, \& Taylor 1995). Unfortunately no measurements of the masses have been obtained to date. However, since all high-precision measurements of the mass of neutron stars are consistent with $1.4 \mathrm{M}_{\odot}$, with very small scatter (Thorsett et al. 1993), it is assumed that the mass of the neutron star in this system is also $1.4 \mathrm{M}_{\odot}$. The formation of binary systems such as this one is sufficiently well understood to allow the determination of $m_{2}$ from other orbital parameters 
(Rappaport et al. 1995; Joss, Rappaport, \& Lewis 1987; Savonije 1987). These methods yield $m_{2}=(0.18 \pm 0.02) \mathrm{M}_{\odot}$, close to the minimum mass of $0.175 \mathrm{M}_{\odot}$ derived from the pulsar mass function

$$
f_{1}\left(m_{1}, m_{2}, i\right)=\frac{\left(m_{2} \sin i\right)^{3}}{\left(m_{1}+m_{2}\right)^{2}}=\frac{4 \pi^{2}\left(a_{1} \sin i\right)^{3}}{G P_{b}^{2}}=0.0022 \mathrm{M}_{\odot},
$$

where $a_{1}$ is the semi-major axis of the pulsar's orbit. A $90 \%$ C.L. upper limit on the mass is $0.45 \mathrm{M}_{\odot}$ (Phinney \& Kulkarni 1994). Using these masses, $i$ can be found from equation 0 .

Given the above parameters for PSR J2317+1439, equations 1 - 3 were evaluated, solving equation 2 numerically. This was done for several values of $m_{2}$ in the range $0.175 \mathrm{M}_{\odot}<m_{2}<0.45 \mathrm{M}_{\odot}$ to test the sensitivity of the limit to changes in $m_{2}$. The resulting upper limits on $\left|\hat{\alpha}_{1}\right|$ are in the range $(1.2-1.7) \times 10^{-4}$, demonstrating that the limit is rather insensitive to the mass of the companion and the inclination of the system. The limit of $\left|\hat{\alpha}_{1}\right|<1.7 \times 10^{-4}(90 \%$ C.L.) is tighter by a factor of 3 than both the previous best limit from pulsar systems of $\left|\hat{\alpha}_{1}\right|<5.0 \times 10^{-4}$ (90\% C.L.; DE92b), and the limit (probing only weak field effects) from planetary data of $\alpha_{1}=(2.1 \pm 3.1) \times 10^{-4}(90 \%$ C.L.; Hellings 1984), which allows an $\alpha_{1}$ as large as $5.2 \times 10^{-4}$.

In principle the eccentricity of binary pulsars can be measured by radio pulse timing techniques to about $\delta e \simeq \sigma /\left(a_{1} \sin i\right)$, where $\sigma$ is the typical uncertainty in the measurement of the absolute arrival time of a pulse. For PSR J2317+1439 $\sigma \approx 1.5 \mu \mathrm{s}$, and $a_{1} \sin i=2.3$ light-sec, consistent with the present measurement precision of $e$. Further improvement on limits for $\hat{\alpha}_{1}$ from binary pulsars is likely to be slow, since $\sigma$ for most pulsars is rather larger than for PSR J2317+1439, and the eccentricity for most is also much larger (Phinney \& Kulkarni 1994). Nevertheless modest improvement can be expected as, over time, the mass, velocity, and especially, eccentricity measurements for PSR J2317+1439 improve. The limit we derived for $\hat{\alpha}_{1}$ further constrains the strong-field regime of theories of gravitation that deviate from general relativity in including extra vector or tensor fields.

We thank Prasenjit Saha for helpful discussions. JFB received support from an Australian Postgraduate Research Award and the Australia Telescope National Facility student program. FC gratefully acknowledges use of NSF grant AST 91-15103 at Princeton, and a fellowship under the auspices of the European Commission while at Jodrell Bank. 


\section{REFERENCES}

Camilo, F., Nice, D. J., \& Taylor, J. H. 1993, ApJ, 412, L37

Camilo, F., Nice, D. J., \& Taylor, J. H. 1995, ApJ. In press

Damour, T. \& Esposito-Farèse, G. 1992a, Class. Quant. Grav., 9, 2093

Damour, T. \& Esposito-Farèse, G. 1992b, Phys. Rev. D, 46, 4128

Damour, T. \& Schäfer, G. 1991, Phys. Rev. Lett., 66, 2549

Damour, T. \& Taylor, J. H. 1992, Phys. Rev. D, 45, 1840

Fixsen, D. J. et al. 1994, ApJ, 420, 445

Hellings, R. W. 1984, in General Relativity and Gravitation, Proceedings of the Tenth International Conference, ed. B. Bertatti, F. de Felice, \& A. Pascolini, Reidel, Dordrecht, 365

Joss, P. C., Rappaport, S., \& Lewis, W. 1987, ApJ, 319, 180

Nordtvedt, K. 1987, ApJ, 320, 871

Phinney, E. S. \& Kulkarni, S. R. 1994, ARAA, 32, 591

Rappaport, S., Podsiadlowski, P., Joss, P. C., DiStefano, R., \& Han, Z. 1995, MNRAS. In press

Savonije, G. J. 1987, Nature, 325, 416

Smoot, G. F. et al. 1991, ApJ, 371, L1

Thorsett, S. E., Arzoumanian, Z., McKinnon, M. M., \& Taylor, J. H. 1993, ApJ, 405, L29

Will, C. M. 1993, Theory and Experiment in Gravitational Physics, (Cambridge: Cambridge University Press) 